\title{
A VISITA DOMICILIAR SOB OLHAR DO USUÁRIO DA ATENÇÃO PRIMÁRIA
}

\author{
THE HOME VISIT UNDER THE PRIMARY ATTENTION USER'S GUIDE \\ Leandro Da Silva Paudarco ${ }^{1}$, Cinoélia Leal de Souza ${ }^{2}$, Elaine Santos da Silva ${ }^{2}$, Denise Lima \\ Magalhães ${ }^{2}$, Kaliane da Silva Paudarco ${ }^{2}$ \\ HGRS ${ }^{1}$, CENTRO UNIVERSITÁRIO UNIFG ${ }^{2}$
}

\begin{abstract}
To analyze the home visit, integrated into the theoretical and practical context of the primary health care of the people, having as study area the municipality in the southwest of Bahia. A qualitative and exploratory investigation was carried out, in which 30 users of the Basic Units of Family Health were interviewed, between February and March 2019, using structured and flexible questionnaires to collect data. To recognize the existence of a dissociation between what is predicted by the theoretical context on the one hand and the experience experienced by primary care users on the other was the central finding of this study, since the home visit approach is centered in the disease, essentially restrictive and limiting a mutualistic dialogue, which contributes negatively in the perceptive formation of its importance to the end users. These results show that home visiting actions call for a re-dimensioning of their method of approach, focused on the promotion of multiprofessional care and with a view to considering the social, cultural, educational, economic and environmental context in which the patient, as well as in the construction of a better theoretical / practical vision of the professionals of the health units.
\end{abstract}

Keywords: Primary Health Care. Visit to Home. Health Centers. Family Health Strategy.

\section{Resumo}

Analisar a visita domiciliar, integrada ao contexto teórico e prático da atenção primária à saúde das pessoas, tendo como área de estudo um município situado no sudoeste da Bahia. Foi realizada uma investigação qualitativa $e$ exploratória, na qual foram entrevistados 30 usuários das Unidades Básicas de Saúde da Família, entre os meses de fevereiro e março de 2019, através do uso de questionários estruturados e flexíveis, para a coleta de dados. Reconhecer a existência de uma dissociação entre aquilo que é preconizado pelo contexto teórico, de um lado, e a experiência vivenciada pelos usuários da atenção primária, do outro, foi a constatação central deste estudo, já que a abordagem da visita domiciliar é centrada na doença, essencialmente restritiva e limitadora de um diálogo mutualístico, o que contribui negativamente na formação perceptiva da sua importância para os usuários finais. Estes resultados mostram que as ações de visita domiciliar clamam por um redimensionamento em seu método de abordagem, centrado na promoção de um atendimento multiprofissional e com vistas a considerar o contexto social, cultural, educacional, econômico e ambiental em que se está inserido o paciente, bem como na construção de uma melhor visão teórico/prática dos profissionais das unidades de saúde.

Palavras-chave: Atenção Primária à Saúde. Visita domiciliar. Unidade Básica de Saúde. Estratégia de Saúde da Família 
A Estratégia de Saúde da Família (ESF) emergiu com foco na reorientação assistencial, voltada para promoção da saúde, dentro da atenção primária. ${ }^{1}$ Com território definido em seu amplo espectro, população adscrita, trabalho em equipe multiprofissional e a intersetoralidade são eixos fundamentais da ESF, trazendo consigo a visita domiciliar como uma de suas ações importantes, objetivando ampliar o acesso aos serviços e criar vínculos com a população. ${ }^{2}$

A visita domiciliar é uma tecnologia que promove a interação no cuidado em saúde, sendo alicerçada no conhecimento das condições da família em seu território compondo um eixo transversal que abarca uma visão holística e que integra a universalidade, a integralidade e a equidade da atenção em saúde baseada nos princípios norteadores do Sistema Único de Saúde (SUS). ${ }^{3}$

O fortalecimento de práticas contra o modelo médico hegemônico e a formulação de novas estratégias e mecanismos para o cuidado em saúde, como a atenção domiciliar, têm sido cada vez mais enfatizado em decorrência da necessidade de transformações sociais espaciais nos últimos anos, o que vem contribuindo para a importância dos laços familiares e sua evolução dentro do seu ambiente de vivência. ${ }^{4}$

Porém, para que se chegasse a atual conjuntura da ESF, muito se foi debatido e reformulado, pois desde os primórdios o cuidado era prestado em domicílios nas classes mais abastadas, enquanto a população base e sem condições, ficava sob a responsabilidade de freiras e demais voluntários em asilos e hospitais filantrópicos. ${ }^{5}$

Para Cunha e Sá ${ }^{6}$ no âmbito nacional, a intervenção domiciliária teve sua apresentação no século $X X$, através de Oswaldo Cruz e outros que buscaram técnicas sanitárias na Europa e implementaram no Brasil, sendo uma das primeiras medidas de saúde coletiva. Nesse período, a visita domiciliar era voltado para o combate de pragas e doenças endêmicas da época que se alastravam pelo estado.

No período de 2006 a 2011 constatou-se uma lacuna e estagnação existente no âmbito da visita domiciliar, o que gerou uma falta de atenção envolvendo a mesma. Para que essa proposta entrasse em ascensão na esfera federal em 2011 o Ministério da Saúde publicou a portaria no 2.029 de agosto, instituindo a Atenção Domiciliar no âmbito do SUS, revogando a portaria anterior de $2006 .^{7}$
Após dois anos o Ministério da Saúde publicou uma nova portaria de no 923, de 27 de maio de 2013, que propôs a redefinição da Atenção Domiciliar no âmbito do SUS, trazendo um novo rumo para a visita domiciliar, dentro de um contexto social abrangente e inovador, caracterizando-se por medidas que propunha mudanças no atual cenário curativista e fragmentador, propondo um novo caminho a saúde, fomentando a sua permanência e consistência, dentro da atenção primária. ${ }^{8}$

Para Rocha, Conz, Barcinski, Paiva e Pizzinato $^{1}$ a visita domiciliar é uma ferramenta itinerante do profissional da atenção primária, como forma de estreitar o vínculo entre comunidade e equipe de saúde, o que permite a inserção e quebra de barreiras existentes entre o coletivo, promovendo um ambiente favorável à junção do conhecimento científico e o popular, atuando primordialmente na saúde coletiva, facilitando a resolução dos problemas de saúde.

Andrade, Guimarães, Costa, Machado e Gois ${ }^{9}$ afirmam que o contexto em que a visita domiciliar está inserida tem refletido cada vez mais o cuidado ao indivíduo, família e comunidades, fundamentada nos preceitos da assistência no território, servindo como forma de alimentar os sistemas de informações em saúde e servir para o planejamento, implementação e avaliação de ações comunitárias.

Para isso as ações incorporadas nesse contexto buscam garantir a promoção da saúde com base nas práticas assistenciais na rede de serviços de saúde que se entrelaçam para melhor qualidade do processo, visando à potencialidade do território e suas conjunturas sociais, econômicas, educacionais, demográficas, ambientais e culturais. ${ }^{10}$

Com base nisso, Savassi et al. ${ }^{11}$ trazem a visita domiciliar como uma tecnologia que vislumbra a compreensão do indivíduo no seu contexto social, familiar e multifatorial, favorecendo o vínculo das relações ambíguas, propõe um dinamismo e traz ações que corroboram para estratégias pedagógicas, sanitárias, assistenciais, ambientais associadas a técnica, mas com uma visão muito além da visão baseada em sintomatologias e diagnósticos. Diante do exposto, este estudo teve como objetivo analisar a visita domiciliar no âmbito teórico e prático a partir da experiência dos usuários da atenção primária à saúde. 


\section{Metodologia}

Tratou-se de uma pesquisa qualitativa exploratória, que de acordo com Cavalcante, Calixto e Pinheiro ${ }^{12}$ tem como características qualificar e descrever as vivências dos sujeitos, bem como sua percepção sobre determinado objeto e seus fenômenos.

Os participantes do estudo foram usuários das Unidades Básicas de Saúde da Família (UBS) de um município, localizado no sudoeste do estado da Bahia, distante 796 quilômetros a sudoeste da capital Salvador, que possui uma população estimada em 2018, segundo o IBGE, ${ }^{13}$ de 84014 habitantes.

A amostra dos participantes foi definida por conveniência e por saturação, que é aquela em que o pesquisador seleciona um determinado número de participantes, e que após aplicações sucessivas, se os dados não acrescentam nenhuma propriedade nova ao campo de estudo, indica o momento em que se deve parar a coleta de dados, permitindo generalizar os resultados para o conjunto ou grupo estudado. ${ }^{14}$ Nesse intuito, após saturação, foram incluídos 30 usuários.

A coleta de dados ocorreu de fevereiro a março de 2019, com os usuários das USF de três bairros, por se tratarem de locais com barreiras socioambientais que, de forma geral, podem ser descritas como falta de saneamento básico, uso de fossas sépticas nos domicílios, bairros com localização próxima a áreas de matas e no que se relaciona ao nível de escolaridade e renda, ambos são baixos. Foi incluído no estudo indivíduos que estavam conscientes, e que aceitaram participar da pesquisa, mediante assinatura do Termo de Consentimento Livre e Esclarecido (TCLE).

$O$ instrumento de coleta de dados foi um roteiro não estruturado e flexível ao andamento da entrevista, composto por 11 questões relacionadas ao perfil socioeconômico dos participantes e 2 questões norteadoras, que versavam sobre as experiências e vivências dos usuários com visitas domiciliares na área de abrangência da ESF, sendo que, cada entrevista durou em média de 20 minutos. Todas as entrevistas foram gravadas perante autorização do participante, para posterior transcrição, assegurando-os quanto à confiabilidade e privacidade dos dados pessoais obtidos.

O tratamento dos dados foi realizado a partir da técnica de análise de conteúdo semântica, seguindo às etapas descritas por $\operatorname{Bardin}^{15}$ e foi dividida em três fases: ordenação dos dados; na qual o material empírico coletado (documentos e transcrições das entrevistas) foi organizado, com a identificação dos participantes por número e a transcrição dos áudios na integra; classificação dos dados e análise propriamente dita. Na segunda etapa foram definidas as unidades de registro: visita domiciliar, domicílio, comunidade e atenção domiciliar.

Na terceira e última fase do tratamento dos dados, foi realizada a análise minuciosa dos resultados, que foram divididos em unidades de registro para a posterior descrição e discussão dos resultados. A partir disso, foram definidas duas categorias de análise e discussão: a visita domiciliar como ferramenta da promoção de saúde na atenção primária; e, a experiência do usuário com a visita domiciliar na atenção primária.

Todas as fases desta pesquisa foram realizadas em consonância com as questões ético-legais da resolução número 466 de 2012, do Conselho Nacional de Saúde que regulamenta as pesquisas envolvendo os seres humanos no Brasil. A pesquisa foi aprovada pelo Comitê de Ética e Pesquisa da UESB campus de Jequié/BA, sob o protocolo CAAE: 79882217.8.0000.0055, em 05 de dezembro de 2017.

\section{Resultados e Discussão}

Foram entrevistados 30 usuários das três UBS. Traçando um perfil sociodemográfico dos participantes do estudo, notou-se que $70 \%$ eram mulheres e $30 \%$ homens; $36 \%$ com idade de 20 a 30 anos, 34\% entre 31 e 50, 13\% entre 61 a 70 e $7 \%$ com idade superior a 70 anos; em relação à situação conjugal $57 \%$ eram casados, $30 \%$ solteiro, $7 \%$ viúvo e $6 \%$ divorciado ou em união estável.

Quanto à escolaridade, $43 \%$ possuíam ensino médio completo, $17 \%$ semianalfabetos, $17 \%$ com ensino fundamental incompleto, $10 \%$ ensino superior incompleto, $7 \%$ ensino fundamental completo, $3 \%$ ensino médio incompleto e 3\% não tinham escolarização. Sobre a renda, 53\% tem renda de 1 salário mínimo, $27 \%$ possuíam renda menor que 1 salário mínimo e 20 $\%$ de 2 a 3 salários mínimos, sendo que destes $57 \%$ não exerciam uma ocupação remunerada e 43\% tinham remuneração.

Segundo Azeredo, Cotta, Schott, Maia e Marques ${ }^{16}$ fatores como a condição de moradia, condições de saneamento, meio socioambiental, nível econômico, educacional e morbidades interferem diretamente na saúde dos indivíduos e da coletividade, e determinará o processo de 
saúde doença e através do conhecimento prévio é possível se intervir para minimizar ou anular os riscos e agravos decorrentes do meio.

Nesta perspectiva, a visita domiciliar tem se tornando um importante método e estratégia para o acompanhamento da terapêutica destinada à doenças, visando educação em saúde por meio da orientação, sensibilização e prevenção de agravos decorrente de patologias de base como a diabetes e hipertensão. $^{17}$

Sendo assim, de acordo com os resultados obtidos através das entrevistas realizadas com os usuários dos serviços de saúde, foram elencadas duas categorias para análises, sendo elas: a visita domiciliar como ferramenta da promoção de saúde na atenção primária; e, a experiência do usuário com a visita domiciliar na atenção primária.

A visita domiciliar como ferramenta da promoção de saúde na atenção primária

$\mathrm{Na}$ avaliação da visita domiciliar, 50\% dos usuários afirmam já ter recebido uma visita domiciliar, e 50\% destes afirmaram que não recebe ou recebeu visita domiciliar, principalmente por ser uma área descoberta ou pouco frequentada pelos Agentes Comunitários de Saúde (ACS) durante as visitas. Nesta perspectiva Barbosa et al. ${ }^{18}$ ao realizar sua pesquisa com 364 usuários de quatro Unidades de Saúde da Família de um município de pequeno porte, cerca de $90 \%$ dos entrevistados gostariam que o ACS fosse em suas residências em uma frequência maior. $E$ destes 50,5\% apontaram a visita do ACS em uma frequência mensal.

Ainda segundo o mesmo autor ${ }^{18}$, a potencialidade expressiva da visita domiciliar reflete diretamente na atuação da atenção primária em seu território favorecendo a compreensão dos seus reais fatores determinantes e condicionantes, atuando em ações diretas e resolutivas destinadas a comunidade, evidenciando principalmente as pessoas que vivem em condições de vulnerabilidade.

Para Magalhães, Giacomin, Santos e Firmo $^{19}$ a visita deve abarcar quatro etapas: planejamento ou organização, execução, registro de dados e avaliação da visita, mas ainda se volta ao indivíduo e não se mostra eficaz na prevenção, educação e intervenção em questões sociais, sendo apenas tecnicista voltada para o acompanhamento empírico, realizada pelos profissionais de saúde atuantes na UBS, quando necessário.

Nesta visão, a população entrevistada afirmou que os profissionais são superficiais em suas visitas, abstendo-se de questões de sensibilização que poderiam colaborar e influenciar na qualidade de vida do familiar, desenvolvendo uma linha de cuidados a saúde em um contexto integralizador e pontecializador de mudanças socioculturais, refletindo na saúde coletiva. Segundo Silva, Arboit, Silveira, Cavalheiro, Krause e Menezes ${ }^{17}$ tais profissionais são peças chaves do quebra cabeça que envolve o território e o processo de saúde doença.

Para Guanaes-Lorenzi, Pinheiro ${ }^{20}$ ao realizar sua pesquisa em uma cidade de pequeno porte no interior de São Paulo, com 28 ACS das seis equipes de saúde da família, enfatizou a importância dos ACS na visita domiciliar e na ESF, por ser a conexão entre a unidade básica e a população facilitando o diálogo social, disposto em sua prática cotidiana, torna-se um recurso fértil construção da ESF de forma ampla e efetiva.

Corroborando com esse estudo, foi evidenciado no decorrer das entrevistas com os usuários um vínculo maior com os ACS durante as visitas, demonstrando uma relação ambígua ao nível interpessoal, proveitosa para um debate livre, consciente, esclarecedor e enriquecedor. Para Pereira, Silva, Santana, Kobayashi e Nogueira $^{21}$ se faz necessário duas visões de mundo para um consenso em comum, e quando não há, interfere no resultado final que é a dissociação da população e a ESF.

Nesse sentido, o ACS se torna indispensável representando o olhar visionário da atenção primária. Neste estudo, 93\% dos participantes responderam que o agente comunitário de saúde realiza as visitas domiciliares, enquanto $7 \%$ responderam que o enfermeiro faz a visita domiciliar. Indo de acordo com a pesquisa de Gomes, Fracolli e Machado ${ }^{22}$ com 100 usuários das unidades da ESF do município de Assis em São Paulo, notou-se que $38 \%$ dos usuários referem que o enfermeiro só faz visita quando solicitado e $82 \%$ afirmam que ACS fazem visitas as suas casas pelo menos uma vez por mês.

A respeito da visita efetuada pelo enfermeiro, percebe-se que esta só é realizada em casos curativistas e por busca do próprio usuário ou familiares, como relatado abaixo.

"Ruim porque não tem, mas era importante ter pois quando tinha marcava exames e consultas. Uma vez a enfermeira veio 
fazer um curativo na minha cirurgia porque minha filha foi buscá-Ia" ( Usuário 1).

Segundo os mesmos autores ${ }^{22}$ a dificuldade do profissional de nível superior, principalmente do enfermeiro em ir à área e as residências dos usuários está associada a sobrecarga do trabalho, afazeres burocráticos presente na unidade e por este profissional ter uma função dupla na ESF, gerencial e assistencial dentro do contexto atual, além da defasagem de profissionais que dificulta o trabalho.

Pereira, Silva, Santana, Kobayashi e Nogueira ${ }^{21}$ criticam o atual modelo das visitas, por ser limitada e se basear apenas ao saber técnico, precisando ser modificado, de maneira que a abordagem à família transcenda o ser biológico, fazendo-se indispensável romper a fragmentação na abordagem do ser humano, articulando novas tecnologias levando em consideração a integralidade. Nesta proposta os usuários veem a visita domiciliar como algo tecnicista e deficiente nas ações educativas, o que torna essas ações restritivas, como relatadas abaixo por um dos usuários.

"Acredito que as visitas a minha casa deveriam ter algo mais, pois a agente só pergunta se estou bem e informa sobre algo do postinho" (Usuário 2).

Destaca-se a importância das visitas dos profissionais de nível superior, pois são evidenciados melhores prognósticos dos pacientes que estão em processo de tratamento e reabilitação. A promoção da integração domiciliar, além do reconhecimento de suas necessidades através da escuta ativa de forma ampliada atua de forma eficaz. ${ }^{23}$

Pesando uma forte concepção do usuário a respeito da rotina da visita domiciliar algo que é necessário, mas que por motivos da rotina do ACS tornou-se pouco significante aos olhos da população. O que segundo De-Carli, Santos, Souza, Kodjaoglanian e Batiston ${ }^{2}$ gera sob a população a insatisfação e desagregação do conceito de promoção em saúde através de ações diversas e multiprofissionais da atenção primária a saúde.

Defendida por Machado, Ferreira e Pinto $^{24}$ a importância do papel da interação da equipe multidisciplinar para uma maior compreensão do ser social, havendo uma integração dos conhecimentos das diversas áreas unidas em um propósito multidisciplinar, possibilitando a realização de um trabalho focado no ser holístico, resgatando o que cada área poderia oferecer de melhor aos seus pacientes e às intervenções no âmbito de promoção através da sensibilização e orientação da família, refletindo em uma melhora expressiva na postura dos componentes.

$\mathrm{Na}$ perspectiva da importância da realização das orientações sobre saúde pelos profissionais que integram a atenção primária, $67 \%$ dos usuários responderam que não realizam tais atividades, enquanto $33 \%$ responderam que recebiam as devidas orientações. Segundo Martins, Guarini, Moreira, Pinheiro, Hatisuka e Junior $^{25}$ as ações desenvolvidas se restringem em acompanhamento e avaliação de idosos com Hipertensão Arterial Sistêmica, Diabetes mellitus, acamados, indivíduos com feridas que carecem da realização de assistência a integridade cutânea, além de estar ligado ao modelo ultrapassado de visão restritiva e apenas curativista, deixando de se abarcar demais conceitos e práticas assistenciais mais ampliadas a integralidade do ser.

Demostrando assim, que há uma descaracterização na visita domiciliar, déficit nas orientações para a promoção de saúde dos usuários da atenção primária, que coopera para carência sob o olhar da população na visita domiciliar, pois esta acaba por se restringir a ações técnicas, que por se tratar de populações em áreas de conflito socioambiental e de um território que exige uma atenção para as questões de saúde coletiva, decorrente da junção de fatores socioculturais, com baixo índice de ações sanitárias torna-se pouco efetiva.

A experiência do usuário com a visita domiciliar na atenção primária

Para Bezerra, Lima e Lima $^{3}$ a visita domiciliar é capaz de contribuir de forma a garantir uma atenção integral a pessoa e o coletivo, através de orientações que abarquem a realidade de saúde do usuário e da concepção de seu modo de vida do conhecimento do ambiente e de suas relações, associado com a propriedade de abarcar questões que abordam o ser em sua mutualidade. Sendo assim, quando questionados os usuários sobre o que é abordado pelos profissionais durante a visita domiciliar, surgiram as seguintes experiências:

"A agente pergunta se houve alguma intercorrência, se há algum problema, além de dizer os dias de atendimento do médico e da enfermeira" (Usuário 3).

"Pergunta como está à saúde, caderneta de vacina, fala do funcionamento do postinho, cuidado com meu bebê e o peso das crianças" (Usuário 4).

"Não tenho agente de saúde e os outros profissionais não vem aqui" (Usuário 5). 
Para Martins, Guarini, Moreira, Pinheiro, Hatisuka e Junior ${ }^{25}$ o potencial da visita domiciliar deveria ir muito além de questões técnicas, não pontuais e sem programação da promoção da saúde, mas sim, ações que proporcionem ao indivíduo a visão deste ser holístico e constituído de diversos fatores e que os profissionais devem considerar como uma atividade sistematizada, obrigatória e importante para programar a adequada assistência.

Matos, Santos, Silva, Del'Sarto e Vieira, 26 enfatizam que dentre os desafios que dificultam a concretização da visita domiciliar está a questão da localização territorial, por existência de vários bairros que a ESF abrange, dificultando o acesso a até a unidade, outro ponto é a relação entre a equipe de saúde, necessitando assim do vínculo interpessoal dos profissionais, além de fatores socioambientais, como pontos de drogas, longas caminhadas de exposição ao sol do ACS, áreas de difícil acesso, sem pavimento e o nível de escolaridade baixo pode interferir na compreensão das orientações fornecidas pelos profissionais.

Também defendido por Fernandes, Duarte e Schmalfuss ${ }^{27}$ que ao realizar a pesquisa com nove profissionais sobre a visita domiciliar, ambos relatam a dificuldade de locomoção até os pacientes, a alta demanda para poucos profissionais, que contribui significativamente para o déficit de visitas, além da alta sobrecarga de trabalho aos que realizam tais ações; outro ponto importante é a questão da qualificação destes profissionais. Para Silva, Arboit, Silveira, Cavalheiro, Krause e Menezes ${ }^{17}$ a qualificação conduz estes profissionais a uma visão mais crítica e reflexiva das reais necessidades e especificidades dos usuários.

Segundo Leonardi ${ }^{28}$, outro ponto para dificuldade da visita domiciliar é o desafio de se construir estratégias para ações que contemplem toda sua complexidade sociocultural, ambiental, econômicas, instrucional e de saúde da população. Neste caminho é primordial a interferência transversal da gestão de equipe, principalmente no quesito de se trabalhar ações modificadoras para surtir efeitos e modificação da realidade.

Torres, Roque e Nunes ${ }^{29}$ afirmam que a visita permite um momento de conexão do profissional da atenção primária com a população, onde o profissional irá abordar temas que sejam pertinentes ao momento, de acordo com a necessidade de cada usuário e ou conforme determinado sistematicamente pela equipe, além de esclarecimento de dúvidas e orientações em geral. Nesta perspectiva quando questionado aos populares quanto aos temas abordados durante as visitas as respostas foram vagas.

(Usuário 6).

"Não me recordo se eles falaram"

"Vacinação, prevenção do câncer de mama e útero, funcionamento da unidade" (Usuário 7).

"Diabetes, hipertensão, vacinação e peso" (Usuário 8).

Para Barbosa et al. ${ }^{18}$ há uma vasta gama de temas e assuntos a serem discutidos na visita domiciliar, entretanto, percebe-se que o foco dos temas foi limitado ao estado geral de saúde, doenças dos usuários e ações pontuais. Em sua pesquisa $89 \%$ dos populares afirmaram que tais questões eram meramente voltadas a temas curativistas e estáticos, com $8 \%$ voltado a orientações sobre consultas e agendamentos.

Mussi et al. ${ }^{30}$ aponta que tais orientações ao indivíduo e família devem ser aplicadas concomitantemente durante os períodos da visita, momento que o profissional se relaciona, conhece e oferece seu conhecimento teórico científico na prática cotidiana do usuário, levando em consideração os vários fatores de interferência e suprindo a necessidade existente no meio que o mesmo está inserido.

Assim, há a necessidade de unir os trabalhos no âmbito de se constituírem uma interação da equipe e principalmente do enfermeiro e do ACS. Não havendo o intercâmbio entre estes profissionais tais atividades e ações ficam fragilizadas, acarretando em práticas de saúde divergentes. Além da anuência de confiança e parceria pode induzir a ocasiões de tensões entre a equipe durante a visita domiciliar. $^{31}$

Já quando questionados os usuários sobre suas opiniões e experiências da visita domiciliar, afirmam que:

"Seria bom ter acompanhamento já que eu sinto desse problema, iria me ajudar muito" (Usuário 9).

"Devia vir alguém do PSF aqui, pois é uma área descoberta" (Usuário 10).

"Os profissionais deveriam interagir muito mais com a comunidade" (Usuário 11).

"Deveria haver uma assistência de qualidade que assistisse a população" (Usuário 12).

Desse modo, tais percepções dos usuários a respeito da visita domiciliar trazem críticas construtivas a mudança do atual modelo constituído está ineficaz e necessita de mudanças e capacitação desta equipe para atuar na linha das necessidades humanas, indo de confronto 
com os resultados de Barbosa et al. ${ }^{18}$ em sua pesquisa realizada com usuários, demostrou que $62,9 \%$ dos entrevistados afirmaram estar parcialmente satisfeitos, enquanto que cerca de um terço encontraram-se satisfeitos.

De acordo com Gaiva, Siqueira ${ }^{32}$ ainda se faz carente de um adequando planejamento e sistematização da visita domiciliar, para contextualizar a assistência dos profissionais a determinadas famílias, grupos alvos e população em geral; promovendo uma autonomia coletiva através do empoderamento com as orientações.

É importante a criação dos vínculos entre os profissionais e família. Para Santos, Morais ${ }^{5}$ esse vínculo tem se mostrado eficiente para a promoção de saúde, decorrente da confiança que o coletivo tem com a equipe de saúde, permitindo um leque de ações em prol do singular ou coletivo, além de conhecer a realidade e compreensão do ser mútuo. Além de fazer com que o usuário passe a atuar de forma ativa e participativa na sua saúde e no coletivo.

\section{Considerações finais}

A visita domiciliar na atenção primária é indispensável, pois empodera os usuários e sensibiliza a população de acordo com cada realidade social, levando em consideração o seu contexto territorial, trabalhando assim com suas percepções. Nesta proposta, o presente estudo refletiu sobre a visita domiciliar ainda ser volta as apenas no âmbito para visão centralizada na patologia e o estado de saúde do indivíduo, deixando de buscar a promoção da saúde através das orientações e vínculo entre profissionais e usuários, além de tornar o ACS em sua maioria os únicos responsáveis pela visita domiciliar. Por isso, é um desafio atual o trabalho dessa equipe no intuito de promover vistas domiciliares multiprofissionais, sendo uma das propostas da ESF.

Portanto, é necessário que a equipe da ESF promova ações a nível descentralizado, trabalhando novas propostas e perspectivas da visita domiciliar e sua importância para a compreensão do ambiente, ser humano e suas relações intrínsecas no intuito de intervir para melhora da visita domiciliar sistematizada e concretizada no âmbito da saúde coletiva. Para isso também é importante à incorporação de novos estudos que complemente o mesmo valorizando os sujeitos subjetivos buscando a integralidade do cuidado.

\section{Referências}

1. Rocha K, Conz J, Barcinski M, PAIVA D, Pizzinato, A. A visita domiciliar no contexto da saúde: uma revisão de literatura. Psicologia, Saúde \& Doenças. 2017;8(1):170-185.

2. De-carli AD, Santos MLM, Souza AS, Kodjaoglanian VL, Batiston AP. Visita domiciliar e cuidado domiciliar na Atenção Básica: Um olhar sobre a saúde bucal. Saúde Debate. 2015;39(105):441-450.

3. Bezerra MIC, Lima MJMR, Lima YCP. A visita domiciliar como ferramenta de cuidado da fisioterapia na Estratégia Saúde da Família. Sanare. 2015;14(01):76-80.

4. Savassi LCM. Os atuais desafios da Atenção Domiciliar na Atenção Primária à Saúde: uma análise na perspectiva do Sistema Único de Saúde. Rev Bras Med Fam Comunidade. 2016;11(38):1-12.

5. Santos EM, Morais SHG. A visita domiciliar na estratégia saúde da família: percepção de enfermeiros. Cogitare Enfermagem. 2011;16(3):492-497.

6. Cunha MS, Sá MC. A visita domiciliar na Estratégia de Saúde da Família: os desafios de se mover no território. Interface - Comunic. Saude Educ. 2013;17(44):61-73.

7. Ministério da Saúde. Caderno de Atenção Domiciliar. 1ed. Brasília: Ministério da Saúde;2012:101.

8. Ministério da Saúde. Caderno de Atenção Domiciliar. 2. ed. Brasília: Ministério da Saúde;2013:204.

9. Andrade AM, Guimarães AMAN, Costa DM, Machado LC, Gois CFL. Visita domiciliar: validação de um instrumento para registro e acompanhamento dos indivíduos e das famílias. Epidemiol. Serv. Saúde. 2014;23(1):165-175.

10. Borges FR, Avelino CCV, Costa LCS, Lourenço DS, Sá MD, Goyatá SLT. Ensino sobre visita domiciliar a estudantes universitários. Rev Rene. 2017;18(1):129-38.

11. Savassi LCM, Melo CGL, Gonçalves DA, Oliveira DOPS, Lopes HR, Fregonezi RLH. Proposta de residência em Atenção Domiciliar como área de atuação. Rev Bras Med Fam Comunidade. 2018;13(40):1-13.

12. Cavalcante RB, Calixto P, Pinheiro MMK. Análise de Conteúdo: considerações gerais, relações com a pergunta de pesquisa, possibilidades e limitações do método. Informação \& Sociedade: Estudos. 2014;24(1):1318.

13. Instituto Brasileiro de Geografia e Estatística [www.ibge.gov.br]. Censo 2010 [Acesso em 08 de março de 2019]. Disponível 
em:

https://cidades.ibge.gov.br/brasil/ba/guanambi

14. Pires ÁP. Sobre algumas questões epistemológicas de uma metodologia geral para as ciências sociais. In: Poupart J. et al. A pesquisa qualitativa: enfoques epistemológicos e metodológicos. Tradução Ana Cristina Nasser. Petrópolis: Editora Vozes;2008.

15. Bardin L. Análise de Conteúdo. 70ed. Lisboa;2011.

16. Azeredo CM, Cotta RMM, Schott $M$, Maia TM, Marques ES. Avaliação das condições de habitação e saneamento: a importância da visita domiciliar no contexto do Programa de Saúde da Família. Ciênc. saúde coletiva. 2007;12(3):743-753.

17. Silva IS, Arboit EL, Silveira MR, Cavalheiro ITF, Krause KMO, Menezes LP. Visita Domiciliar: Estratégia para a promoção da saúde de pacientes crônicos. Revista de Enfermagem. 2016;12(12):88-99.

18. Barbosa DCM, Mattos ATR, Corrêa MH, Faria M, Ribeiro LC, Santos LL, Ferreira JBB, Forster AC. Visita domiciliar sob a percepção dos usuários da ESF. Medicina (Ribeirão Preto). 2016;49(4):360-366.

19. Magalhães KA, Giacomin KC, Santos WJ, Firmo JOA. A visita domiciliária do agente comunitário de saúde a famílias com idosos frágeis. Ciência \& Saúde Coletiva. 2015;20(12):3787-3796.

20. Guanaes-lorenzi C, Pinheiro RLA. (des)valorização do agente comunitário de saúde na Estratégia Saúde da Família. Ciência \& Saúde Coletiva. 2016;21(8):2537-2546.

21. Pereira CEA, Silva MVS, Santana ME, Kobayashi DR, Nogueira LMV. O reflexo da visita domiciliar do ACS na busca ativa do SR de um município da Amazônia. Rev. APS. 2018;21(1):77 -85 .

22. Gomes MFP, Fracolli LA, Machado BC. Atenção domiciliar do enfermeiro na estratégia saúde da família. O Mundo da Saúde. 2015;39(4):470-475.

23. Andrade AM, Silva KL, Seixas CT, Braga PP. Nursing practice in home care: an integrative literature review. Rev Bras Enferm [Internet]. 2017;70(1):199-208.

DOI: http://dx.doi.org/10.1590/0034-7167-2016-0214 24. Machado EE, Ferreira RPC, Pinto TC. Papel da equipe multidisciplinar em nível de atenção domiciliar. Revista Cadernos de Educação, Saúde e Fisioterapia. 2018;5(10):111111. https://doi.org/10.18310/2358-8306

25. Martins AB, Guarini G, Moreira RC, Pinheiro EFC, Hatisuka MFB, Junior CSG. Perfil sociodemográfico e as ações da equipe de atenção básica em saúde do idoso. Brazilian Journal of Surgery and Clinical Research - BJSCR. 2018;22(2):07-13.

26. Matos DS, Santos AT, Silva JM, Del'sarto RBO, Vieira SNS. Visita domiciliar: dificuldades encontradas pelos Agentes Comunitários de Saúde. EFDeportes.com [Revista Digital] 2016 [acessado em 02 de abril de 2019] 20(214). Disponível em: https://www.efdeportes.com/efd214/visitadomiciliar-dificuldades-pelos-acs.htm

27. Fernandes M, Duarte MLC, Schmalfuss JM. Facilidades e dificuldades na realização de visitas domiciliares em um centro de atenção psicossocial. Cogitare Enferm. 2014;19(3):451458.

28. Leonardi, F. G. Visitas domiciliares: desafio à atenção básica e saúde mental. Cadernos Brasileiros de Saúde Mental, Florianópolis. 2018;10(26):01-18.

29. Torres HC, Roque C, Nunes C. Visita domiciliar: estratégia educativa para $\mathrm{O}$ autocuidado de clientes diabéticos na atenção básica. Rev. enferm. UERJ. 2011;19(1):89-93.

30. Mussi CM, Ruschel K, Souza EN, Lopes ANM, Trojahn MM, Paraboni CC, Rabelo ER. Visita domiciliar melhora conhecimento, autocuidado e adesão na insuficiência cardíaca: ensaio clínico randomizado HELEN-I1. Rev. Latino-Am. Enfermagem [Internet]. jan.-fev. 2013 [acesso em: 04 de abril de 2019];21(Spec):[09 telas]. Disponível em: http://www.scielo.br/pdf/rlae/v21nspe/pt_04.pd f

31. Kebian LVA, Acioli S. A visita domiciliar de enfermeiros e agentes comunitários de saúde da Estratégia Saúde da Família. Rev. Eletr. Enf. 2014;16(1):161-169.

32. Gaíva MAM, Siqueira VCA. Visita Domiciliária pelos profissionais da Estratégia Saúde da Família. Cienc Cuid Saude. 2011;10(4):697-704. 


\section{Endereço para Correspondência}

Leandro Da Silva Paudarco

Endereço: Rua Silveira Martins, S/N.

Bairro: Cabula

Salvador - Bahia

CEP: $41.150-000$

E-mail: leandropaudarco@hotmail.com

Recebido em 02/12/2020

Aprovado em 08/12/2021

Publicado em 30/12/2021 